\title{
Some Public Tripled Coincidence Fixed Point Theorems for \\ Continuous Mappings having Public Mixed $g$ _Monotone Property
}

\author{
Zena Hussein Maibed \\ Department of Mathematics, College of Education for Pure Science, \\ Ibn Al-Haithem, University of Baghdad
}

\section{Abstract}

In this paper, we introduce the pubic fixed point, public coincidence point,pubic mixed g_ monotone propertyand public commute.Also, we proved theexistence and uniqueness of public coincidence fixed point and public fixed point for continuous mappings having public mixed g_monotone property without public commute in partially ordered metric space

Keywords:tripledfixed point, tripledcoincidence point, mixed g_ monotone property

\section{Introduction}

The existence of fixed point for contraction type in partially ordered metric space was first considered by Ran and Reurings[1], they established new results in partially ordered metric space .Also , many researchers presented new results for contraction mappingin partially ordered metricspace see([2]-[25] ). In 2006, Bhaskar and Lakshmikantham [5] introduced the concept mixed monotone property for contractive mappings they also established coupled fixed results for mappings has mixed monotone property.On other hand ,Sintunavarate et al. ([26], [27]) proved the existence and uniqueness of coupled fixed point theorem for nonlinear contractions without the mixed monotone property.lso ,Lakshmikantham and cric [12] introduced the concept of mixed g_-monotone and proved some results for coupled coincidence fixed point and coupled common fixed point for commuting mappings, thisresults extend of results Bhaskar and Lakshmikantham[5].

Additionally ,Choudhury and Kundu[6] ,introduced the compatibity of mappings in partially ordered metric space and they established a coupled coincidence point results.Berinde and Borcut([28], [29]) introduced the concept of tripled fixed point and tripled coincidence fixed point ,they extended the results of Bhaskar and Lakshmikantham[5] and Ciric and Lakshmikantham[12] to the tripled fixed point and coincidence fixed point.

Now, we recall the flowing definitions:

Definition (1.1): [30]

$A$ set $X$ with a binary operation $\leq$ is called partially ordered set if for all $p, q, r \in X$.

i. $p \leq p$

ii. $\quad p \leq q$ and $q \leq p \Rightarrow p=q$

iii. $\quad p \leq q$ and $q \leq r \Rightarrow p \leq r$ 


\section{Definition (1.2): [28]}

Let $f: X^{3} \rightarrow X$ be any mapping. An element $(x, y, z) \in X^{3}$ is called tripled fixed point of $f$ iff $f_{(x, y, z)}=x \quad \& f_{(y, x, y)}=y \quad$ and $f_{(z, y, x)}=z$

\section{Definition (1.3): [29]}

$X$ and $g: X \rightarrow X$ be two mapping. An element $(x, y, z)$ is called a tripled coincidence point of $f$ and $g$ if,

$f_{(x, y, z)}=g_{(x)} \& f_{(y, x, y)}=g_{(y)} \quad$ and $\quad f_{(z, y, x)}=g_{(z)}$

\section{Definition (1.4): [29]}

Let $f: X^{3} \rightarrow X$ and $g: X \rightarrow X$ be two mapping and $(X, \leq)$ be a parlially ordered set, then we Say that $f$ has mixed $\mathrm{g}$ - monotone property if $f$ is monotone increasing in $x$ and $z$ and is monotone decreasing in $y$, i.e, $\forall x, y, z \in X$

$x_{1}, x_{2} \in X, g_{\left(x_{1}\right)} \leq g_{\left(x_{2}\right)} \rightarrow f_{\left(x_{1}, y, z\right)} \leq f_{\left(x_{2}, y, z\right)}$

$y_{1}, y_{2} \in X, g_{\left(y_{1}\right)} \leq g_{\left(y_{2}\right)} \rightarrow f_{\left(x, y_{1}, z\right)} \leq f_{\left(x, y_{2}, z\right)}$

Andz $z_{1}, z_{2} \in X, g_{\left(z_{1}\right)} \leq g_{\left(z_{2}\right)} \rightarrow f_{\left(x, y, z_{1}\right)} \leq f_{\left(x, y, z_{2}\right)}$.

Now, we will give the following concepts

\section{Definition (1.5):}

Let $X$ be a nonempty set. Then we say that the mappings

$f_{1}, f_{2}, \ldots \ldots \ldots f_{n}: X^{3} \rightarrow X$ and $g: X \rightarrow X$ are public commuting if for each $x, y, z \in X$,

$\left(g\left(f_{1}\left(f_{2}\left(\ldots \ldots\left(f_{n(x, y, z)}\right) \ldots \ldots\right)\right)\right)\right)=f_{1}\left(f_{2}\left(\ldots \ldots\left(f_{n(g(x), g(y), g(z))}\right) \ldots \ldots\right)\right)$

\section{Definition (1.6):}

Let $X$ be a nonempty and $f_{1}, f_{2}, \ldots, f_{2}: X^{3} \rightarrow X$ be a given mappings. An element $(x, y, z) \in X^{3}$ is called a public tripled fixed point of $f_{1}, f_{2}, \ldots \ldots, f_{n}$ if

$f_{1}\left(f_{2}\left(\ldots \ldots\left(f_{n(x, y, z)}\right) \ldots \ldots\right)\right)=x, f_{1}\left(f_{2}\left(\ldots \ldots\left(f_{n(y, x, z)}\right) \ldots \ldots\right)\right)=y$ and

$f_{1}\left(f_{2}\left(\ldots \ldots\left(f_{n(z, y, x)}\right) \ldots \ldots\right)\right)=z$

\section{Definition (1.7):}

Let $(X, \leq)$ be a partially ordered set and $f_{1}, f_{2}, \ldots \ldots, f_{n}: X^{3} \rightarrow X$ be a mapping. We say that $f_{1}, f_{2}, \ldots \ldots, f_{n}$ are public mixed monotone if $f_{1}\left(f_{2}\left(\ldots \ldots\left(f_{n(x, y, z)}\right) \ldots \ldots\right)\right)$ is monotone increasing in $x$ and $z$ and is monotone decreasing in $y$, i.e,

$\forall x, y, z \in X$

$x_{1}, x_{2} \in X, x_{1} \leq x_{2} \rightarrow f_{1}\left(f_{2}\left(\ldots\left(f_{n\left(x_{1}, y, z\right)}\right) \ldots\right)\right) \leq f_{1}\left(f_{2}\left(\ldots\left(f_{n\left(x_{2}, y, z\right)}\right) \ldots\right)\right)$

$y_{1}, y_{2} \in X, y_{1} \leq y_{2} \rightarrow f_{1}\left(f_{2}\left(\ldots\left(f_{n\left(x, y_{1}, z\right)}\right) \ldots\right)\right) \geq f_{1}\left(f_{2}\left(\ldots\left(f_{n\left(x, y_{2}, z\right)}\right) \ldots\right)\right)$

And

$z_{1}, z_{2} \in X, z_{1} \leq z_{2} \rightarrow f_{1}\left(f_{2}\left(\ldots\left(f_{n\left(x, y, z_{1}\right)}\right) \ldots\right)\right) \leq f_{1}\left(f_{2}\left(\ldots\left(f_{n\left(x, y, z_{2}\right)}\right) \ldots\right)\right)$ 


\section{Definition (1.8):}

Let $X$ be a nonempty set. Let $f_{1}, f_{2}, \ldots \ldots, f_{3}: X^{3} \rightarrow X$ and $g: X \rightarrow X$ are mappings. An element $(x, y, z) \in X^{3}$ is called public tripled coincidence point of $f_{1}, f_{2}, \ldots, f_{n}$ and $g$ if, $f_{1}\left(f_{2}\left(\ldots\left(f_{n(x, y, z)}\right) \ldots\right)\right)=g(x), f_{1}\left(f_{2}\left(\ldots\left(f_{n(y, x, y)}\right) \ldots\right)\right)=g(y)$

$f_{1}\left(f_{2}\left(\ldots\left(f_{n(z, y, x)}\right) \ldots\right)\right)=g(z)$.

\section{Definition (1.9):}

Let $(X, \leq)$ be a partially ordered set and $f_{1}, f_{2}, \ldots \ldots, f_{n}: X^{3} \rightarrow X$ and $g: X \rightarrow X$ are mappings, then we say that $f_{1}, f_{2}, \ldots \ldots, f_{n}$ are public mixed $g \_$monotone property, If

$f_{1}\left(f_{2}\left(\ldots\left(f_{n(x, y, z)}\right) \ldots\right)\right)$ is monotone increasing in $x$ and $z$ and is monotone decreasing in $y$; i.e, for all $x, y, z \in X$

$x_{1}, x_{2} \in X, g\left(x_{1}\right) \leq g\left(x_{2}\right) \Longrightarrow$

$f_{1}\left(f_{1}\left(\ldots \ldots\left(f_{n\left(x_{1}, y, z\right)}\right) \ldots \ldots\right)\right) \leq f_{1}\left(f_{2}\left(\ldots \ldots\left(f_{n\left(x_{2}, y, z\right)}\right) \ldots \ldots\right)\right)$

$y_{1}, y_{2} \in X, g\left(y_{1}\right) \leq g\left(y_{2}\right) \Rightarrow$

$f_{1}\left(f_{1}\left(\ldots \ldots\left(f_{n\left(x, y_{1}, z\right)}\right) \ldots \ldots\right)\right) \geq f_{1}\left(f_{2}\left(\ldots \ldots\left(f_{n\left(x, y_{2}, z\right)}\right) \ldots \ldots\right)\right)$

Also

$z_{1}, z_{2} \in X, g\left(z_{1}\right) \leq g\left(z_{2}\right) \Rightarrow$

$f_{1}\left(f_{1}\left(\ldots \ldots\left(f_{n\left(x, y, z_{1}\right)}\right) \ldots \ldots\right)\right) \geq f_{1}\left(f_{2}\left(\ldots \ldots\left(f_{n\left(x, y, z_{2}\right)}\right) \ldots \ldots\right)\right)$.

\section{Main Results}

Consider, $\emptyset$ be the set of all increasing mappings such that:

$\emptyset_{i}:[0, \infty] \rightarrow[0, \infty]$ such that $\emptyset_{i(t)}= \begin{cases}t & \text { if } t>0 \\ 0 & \text { if } t<0\end{cases}$

and $\lim _{n \rightarrow \infty} \emptyset_{i}{ }^{n}(t)=0 \forall i=1, \ldots, 7$, where $\emptyset^{n}$ denotes the $\mathrm{n}-$ th iterate of $\emptyset$. But $\mathcal{M}$ is the set of all mappings $f_{1}, \ldots \ldots, f_{n}$ and $g$ satisfy:

i. $g(X)$ is complete of $X$ containing $f_{1}\left(f_{2}\left(\ldots \ldots\left(f_{n}(X \times X \times X)\right) \ldots \ldots\right)\right)$.

ii. $g$ is continuous mappings.

iii. $f_{1}, f_{2}, \ldots \ldots, f_{n}$ are public mixed $g_{-}$monotone property

Now, we prove the following results

\section{Theorem (2.1):}

Let $(X, d, \leq)$ be a partially ordered complete metric space and let $f_{1}, f_{2}, \ldots \ldots, f_{n}: X^{3} \rightarrow$ $X \& g_{1}, g_{2}, \ldots \ldots, g_{n}: X \rightarrow X$ are mappings lies in $\mathcal{M}$. If $\emptyset_{1}, \emptyset_{2}, \ldots \ldots, \emptyset_{7} \in \phi$ such that

$d f_{1}\left(f_{2}\left(\ldots \ldots\left(f_{n(x, y, z)}\right) \ldots \ldots\right)\right), f_{1}\left(f_{2}\left(\ldots \ldots\left(f_{n(u, v, w)}\right) \ldots \ldots\right)\right) \leq$

$\max \left(\emptyset_{1} d(g(x), g(u)), \emptyset_{2} d(g(y), g(v)), \emptyset_{3} d(g(z), g(w)), \emptyset_{4} d\left(f_{1}\left(f_{2}\left(\ldots \ldots\left(f_{n(x, y, z)}\right) \ldots \ldots\right)\right), g(x)\right)\right.$,

$\emptyset_{5} d\left(f_{1}\left(f_{2}\left(\ldots \ldots\left(f_{n(z, y, x)}\right) \ldots \ldots\right)\right), g(z)\right), \emptyset_{6} d\left(f_{1}\left(f_{2}\left(\ldots \ldots\left(f_{n(u, v, w)}\right) \ldots \ldots\right)\right), g(u)\right)$,

$\left.\emptyset_{7} d\left(f_{1}\left(f_{2}\left(\ldots \ldots\left(f_{n(w, v, u)}\right) \ldots \ldots\right)\right), g(w)\right)\right\}$ 
For all $x, y, z, u, v, w \in X$ with

$g(x) \geq g(u), g(y) \leq g(v), g(z) \leq g(w)$

Suppose that, if $\left\langle x_{n}>\right.$ increasing sequence such that: $x_{n} \rightarrow x$ then $x_{n} \leq x$ for all $n \in N$.Also, if $<y_{n}>$ decreasing sequence such that:

$y_{n} \rightarrow y$ then $y_{n} \geq y$ for all $n \in N$.If there exit $x_{\circ}, y_{\circ}, z_{\circ} \in X$ such that:

$$
\begin{aligned}
& g\left(x_{o}\right) \leq f_{1}\left(f_{2}\left(\ldots \ldots\left(f_{n\left(x_{o}, y_{o}, z_{o}\right)}\right) \ldots \ldots\right)\right) \\
& g\left(y_{o}\right) \geq f_{1}\left(f_{2}\left(\ldots \ldots\left(f_{n\left(y_{o}, x_{o}, y_{o}\right)}\right) \ldots \ldots\right)\right)(2.1) \\
& g\left(z_{o}\right) \leq f_{1}\left(f_{2}\left(\ldots \ldots\left(f_{n\left(z_{o}, y_{o}, x_{o}\right)}\right) \ldots \ldots\right)\right)
\end{aligned}
$$

Then $f_{1}, f_{2}, \ldots \ldots, f_{n}$ and $g$ having public tripled coincidence point.

\section{Proof:}

consider $x_{o}, y_{o}, z_{o} \in \in X$ satisfy (2.1). We can construct sequences as,

Define: $g\left(x_{1}\right) \leq f_{1}\left(f_{2}\left(\ldots \ldots\left(f_{n\left(x^{\circ}, y^{\circ}, z^{\circ}\right)}\right) \ldots \ldots\right)\right) g\left(y_{1}\right) \leq f_{1}\left(f_{2}\left(\ldots \ldots\left(f_{n\left(y_{\circ}, x_{\circ}, y_{\circ}\right)}\right) \ldots \ldots\right)\right)$

$$
g\left(z_{1}\right) \leq f_{1}\left(f_{2}\left(\ldots \ldots\left(f_{n\left(z_{0}, y_{0}, x_{0}\right)}\right) \ldots \ldots\right)\right)
$$

And hence, we get:

$$
g\left(x_{0}\right) \leq g\left(x_{1}\right), g\left(y_{0}\right) \geq g\left(y_{1}\right), g\left(z_{0}\right) \leq g\left(z_{1}\right)
$$

As the same way define

$$
\begin{aligned}
& g\left(x_{2}\right)=f_{1}\left(f_{2}\left(\ldots \ldots\left(f_{n\left(x_{1}, y_{1}, z_{1}\right)}\right) \ldots \ldots\right)\right) \\
& g\left(y_{2}\right)=f_{1}\left(f_{2}\left(\ldots \ldots\left(f_{n\left(y_{1}, x_{1}, y_{1}\right)}\right) \ldots \ldots\right)\right) \\
& g\left(z_{2}\right)=f_{1}\left(f_{2}\left(\ldots \ldots\left(f_{n\left(z_{1}, y_{1}, x_{1}\right)}\right) \ldots \ldots\right)\right)
\end{aligned}
$$

But $f_{1}, f_{2}, \ldots \ldots, f_{n}$ having public $g_{-}$mixed monotone property.Then, we get

$$
g\left(x_{0}\right) \leq g\left(x_{1}\right) \leq g\left(x_{2}\right)
$$

$g\left(y_{0}\right) \geq g\left(y_{1}\right) \geq g\left(y_{2}\right)$

$g\left(z_{0}\right) \leq g\left(z_{1}\right) \leq g\left(z_{2}\right)$

We continue operations where we get the sequences.

$<g\left(x_{n}\right)>,<g\left(y_{n}\right)>$ and $<g\left(z_{n}\right)>$ in $g(X)$ and satisfy the following

$$
\begin{aligned}
& g\left(x_{n}\right)=f_{1}\left(f_{2}\left(\ldots \ldots\left(f_{n\left(x_{n-1}, y_{n-1}, z_{n-1}\right)}\right) \ldots \ldots\right)\right) \text {, } \\
& \leq g\left(x_{n+1}\right)=f_{1}\left(f_{2}\left(\ldots \ldots\left(f_{n}\left(x_{n}, y_{n}, z_{n}\right)\right) \ldots \ldots\right)\right)
\end{aligned}
$$$$
g\left(y_{n+1}\right)=f_{1}\left(f_{2}\left(\ldots \ldots\left(f_{n\left(y_{n}, x_{n}, y_{n}\right)}\right) \ldots \ldots\right)\right) \text {, }
$$$$
\leq g\left(y_{n}\right)=f_{1}\left(f_{2}\left(\ldots \ldots\left(f_{n\left(y_{n-1}, x_{n-1}, y_{n-1}\right)}\right) \ldots \ldots\right)\right)
$$$$
g\left(z_{n}\right)=f_{1}\left(f_{2}\left(\ldots \ldots\left(f_{n\left(z_{n-1}, y_{n-1}, x_{n-1}\right)}\right) \ldots \ldots\right)\right) \text {, }
$$$$
\leq g\left(z_{n+1}\right)=f_{1}\left(f_{2}\left(\ldots \ldots\left(f_{n\left(z_{n}, y_{n}, x_{n}\right)}\right) \ldots \ldots\right)\right)
$$

We will take two cases during the proof

Case (1): If,

$$
\left(g\left(x_{n+1}\right), g\left(y_{n+1}\right), g\left(z_{n+1}\right)\right)=\left(g\left(x_{n}\right), g\left(y_{n}\right), g\left(z_{n}\right)\right)
$$

For some $n \in \mathbb{N}$, then 
$f_{1}\left(f_{2}\left(\ldots \ldots\left(f_{n\left(x_{n}, y_{n}, z_{n}\right)}\right) \ldots \ldots\right)\right)=g\left(x_{n}\right)$

$f_{1}\left(f_{2}\left(\ldots \ldots\left(f_{n\left(y_{n}, x_{n}, y_{n}\right)}\right) \ldots \ldots\right)\right)=g\left(y_{n}\right)$

$f_{1}\left(f_{2}\left(\ldots \ldots\left(f_{n\left(z_{n}, y_{n}, x_{n}\right)}\right) \ldots \ldots\right)\right)=g\left(z_{n}\right)$

Hence, $\left(x_{n}, y_{n}, z_{n}\right)$ is a public tripled coincidence point of $f_{1}, f_{2}, \ldots \ldots, f_{n}$ and $g$

\section{Case (2):If}

$$
\left(g\left(x_{n+1}\right), g\left(y_{n+1}\right), g\left(z_{n+1}\right)\right) \neq\left(g\left(x_{n}\right), g\left(y_{n}\right), g\left(z_{n}\right)\right)
$$

$\forall n \in N$, either $g\left(x_{n}\right) \neq g\left(x_{n+1}\right)$ Or $g\left(y_{n}\right) \neq g\left(y_{n+1}\right)$ Or $g\left(z_{n}\right) \neq g\left(x_{n+1}\right)$

Now,

$d\left(g\left(x_{n+1}\right), g\left(x_{n}\right)\right)$

$=d\left(f_{1}\left(f_{2}\left(\ldots \ldots\left(f_{n\left(x_{n}, y_{n}, z_{n}\right)}\right) \ldots \ldots\right)\right), f_{1}\left(f_{2}\left(\ldots \ldots\left(f_{n\left(x_{n-1}, y_{n-1}, z_{n-1}\right)}\right) \ldots \ldots\right)\right)\right)$

$\leq \max \phi_{1} d\left(g\left(x_{n}\right), g\left(x_{n-1}\right)\right), \emptyset_{2} d\left(g\left(y_{n}\right), g\left(y_{n-1}\right)\right), \emptyset_{3} d\left(g\left(z_{n}\right), g\left(z_{n-1}\right)\right), \emptyset_{4} d\left(f_{1}\left(f_{2}\left(\ldots \ldots\left(f_{n\left(x_{n}, y_{n}, z_{n}\right)}\right)\right.\right.\right.$

$$
\begin{gathered}
\emptyset_{7} d\left(f_{1}\left(f_{2}\left(\ldots \ldots\left(f_{n\left(z_{n}, y_{n}, x_{n}\right)}\right) \ldots \ldots\right)\right), g\left(z_{n}\right)\right), \\
\emptyset_{6} d\left(f_{1}\left(f_{2}\left(\ldots \ldots\left(f_{n\left(x_{n-1}, y_{n-1}, z_{n-1}\right)}\right) \ldots \ldots\right)\right), g\left(x_{n-1}\right)\right), \\
\left.\emptyset_{7} d\left(f_{1}\left(f_{2}\left(\ldots \ldots\left(f_{n\left(z_{n-1}, y_{n-1}, x_{n-1}\right)}\right) \ldots \ldots\right)\right), g\left(z_{n-1}\right)\right)\right\}
\end{gathered}
$$

$=$

$$
\begin{aligned}
& \begin{array}{l}
\max \left\{\emptyset_{1} d\left(g_{1}\left(g_{2}\left(\ldots \ldots\left(g_{n\left(x_{n}\right)}\right) \ldots \ldots\right)\right), g\left(x_{n-1}\right)\right), \emptyset_{2} d\left(g\left(y_{n}\right), g\left(y_{n-1}\right)\right), \emptyset_{3} d\left(g\left(z_{n}\right), g\left(z_{n-1}\right.\right.\right. \\
), \emptyset_{7} d\left(g\left(z_{n}\right), g\left(z_{n-1}\right)\right)\right\} \\
\text { Let } h_{1(t)}=\max \left(\emptyset_{1(t)}, \emptyset_{6(t)}\right\}, h_{2(t)}=\max \left(\emptyset_{3(t)}, \emptyset_{7(t)}\right\}, \text { and } \emptyset_{(t)} \in \phi \\
\quad=\max \left(h_{1} d\left(g\left(x_{n}\right), g\left(x_{n-1}\right)\right), \emptyset_{2} d\left(g\left(y_{n}\right), g\left(y_{n-1}\right)\right), h_{2} d\left(g\left(z_{n}\right), g\left(z_{n-1}\right)\right)\right. \text {, }
\end{array} \\
& \left.\emptyset_{4} d\left(g\left(x_{n+1}\right), g\left(x_{n}\right)\right), \emptyset_{5} d\left(g\left(z_{n+1}\right), g\left(z_{n}\right)\right)\right\} \\
& \leq \max \left(h_{1} d\left(g\left(x_{n}\right), g\left(x_{n-1}\right)\right), \emptyset_{2} d\left(g\left(y_{n}\right), g\left(y_{n-1}\right)\right)\right. \text {, } \\
& h_{2} d\left(g\left(z_{n}\right),\left(z_{n-1}\right)\right), \emptyset_{4} d\left(g\left(x_{n+1}\right), g\left(x_{n}\right)\right) \text {, } \\
& \left.\emptyset d\left(g\left(y_{n+1}\right), g\left(y_{n}\right)\right), \emptyset_{5} d\left(g\left(z_{n+1}\right), g\left(z_{n}\right)\right)\right\}
\end{aligned}
$$

Now,

$$
\begin{gathered}
d\left(g\left(y_{n}\right), g\left(y_{n+1}\right)\right) \\
=d\left(f_{1}\left(f_{2}\left(\ldots \ldots\left(f_{n\left(y_{n-1}, x_{n-1}, y_{n-1}\right)}\right) \ldots \ldots\right)\right), f_{1}\left(f_{2}\left(\ldots \ldots\left(f_{n\left(y_{n}, x_{n}, y_{n}\right)}\right) \ldots \ldots\right)\right)\right) \\
\leq \max \left(h_{3} d\left(g\left(y_{n-1}\right), g\left(y_{n}\right)\right), \emptyset_{2} d\left(g\left(x_{n-1}\right), g\left(x_{n}\right)\right),\right. \\
\\
h_{4} d\left(f_{1}\left(f_{2}\left(\ldots \ldots\left(f_{n\left(y_{n-1}, x_{n-1}, y_{n-1}\right)}\right) \ldots \ldots\right)\right), g\left(y_{n-1}\right)\right), \\
\\
\left.h_{5} d\left(f_{1}\left(f_{2}\left(\ldots \ldots\left(f_{n\left(y_{n}, x_{n}, y_{n}\right)}\right) \ldots \ldots\right)\right), g\left(y_{n}\right)\right)\right\}
\end{gathered}
$$

where, $h_{3(t)}=\max \left\{\varnothing_{1(t)}, \emptyset_{3(t)}\right\}, h_{4(t)}=\max \left\{\varnothing_{4(t)}, \varnothing_{5(t)}\right\}$,

$h_{5(t)}=\max \left\{\varnothing_{6(t)}, \emptyset_{7(t)}\right\}, h_{6(t)}=\max \left\{h_{3(t)}, h_{4(t)}\right\}$ and $\emptyset_{(t)} \in \phi$ $\left.d\left(g\left(y_{n}\right), g\left(y_{n+1}\right)\right)=\max _{(2} h_{6} d\left(g\left(y_{n-1}\right), g\left(y_{n}\right)\right), \emptyset_{2} d\left(g\left(x_{n-1}\right), g\left(x_{n}\right)\right), h_{5} d\left(g\left(y_{n+1}\right), g\left(y_{n}\right)\right)\right\}$ 


\section{$\leq \max _{2} d\left(g\left(x_{n-1}\right), g\left(x_{n}\right)\right), h_{6} d\left(g\left(y_{n-1}\right), g\left(y_{n}\right)\right), \emptyset d\left(g\left(z_{n-1}\right), g\left(z_{n}\right)\right)$,}

$\left.\emptyset d\left(g\left(x_{n+1}\right), g\left(x_{n}\right)\right), h_{5} d\left(g\left(y_{n+1}\right), g\left(y_{n}\right)\right), \emptyset d\left(g\left(z_{n+1}\right), g\left(z_{n}\right)\right)\right\}$

Also we have:

$$
\begin{gathered}
d\left(g\left(z_{n+1}\right), g\left(z_{n}\right)\right) \\
=d\left(f_{1}\left(f_{2}\left(\ldots \ldots\left(f_{n\left(z_{n}, y_{n}, x_{n}\right)}\right) \ldots \ldots\right)\right), f_{1}\left(f_{2}\left(\ldots \ldots\left(f_{n\left(z_{n-1}, y_{n-1}, x_{n-1}\right)}\right) \ldots \ldots\right)\right)\right) . \\
\leq \max \left(\emptyset_{1} d\left(g\left(z_{n}\right), g\left(z_{n-1}\right)\right), \emptyset_{2} d\left(g\left(y_{n}\right), g\left(y_{n-1}\right)\right),\right. \\
\emptyset_{3} d\left(g\left(x_{n}\right), g\left(x_{n-1}\right)\right), \emptyset_{4} d\left(f_{1}\left(f_{2}\left(\ldots \ldots\left(f_{n\left(z_{n}, y_{n}, x_{n}\right)}\right) \ldots \ldots\right)\right), g\left(z_{n}\right)\right), \\
\emptyset_{5} d\left(f_{1}\left(f_{2}\left(\ldots \ldots\left(f_{n\left(x_{n}, y_{n}, z_{n}\right)}\right) \ldots \ldots\right)\right), g\left(x_{n}\right)\right), \\
\emptyset_{6} d\left(f_{1}\left(f_{2}\left(\ldots \ldots\left(f_{n\left(z_{n-1}, y_{n-1}, x_{n-1}\right)}\right) \ldots \ldots\right)\right), g\left(z_{n-1}\right)\right), \\
\left.\emptyset_{7} d\left(f_{1}\left(f_{2}\left(\ldots \ldots\left(f_{n\left(x_{n-1}, y_{n-1}, z_{n-1}\right)}\right) \ldots \ldots\right)\right), g\left(x_{n-1}\right)\right)\right\} . \\
=\max \left(\emptyset_{1} d\left(g\left(z_{n}\right), g\left(z_{n-1}\right)\right), \emptyset_{2} d\left(g\left(y_{n}\right), g\left(y_{n-1}\right)\right),\right. \\
\emptyset_{3} d\left(g\left(x_{n}\right), g\left(x_{n-1}\right)\right), \emptyset_{4} d\left(g\left(z_{n+1}\right), g\left(z_{n}\right)\right), \\
\emptyset_{5} d\left(g\left(x_{n+1}\right), g\left(x_{n}\right)\right), \emptyset_{6} d\left(g\left(z_{n}\right), g\left(z_{n-1}\right)\right), \\
\left.\emptyset_{7} d\left(\left(x_{n}\right), g\left(x_{n-1}\right)\right)\right\} . \\
\leq \max \left(h_{7} d\left(g\left(z_{n}\right), g\left(z_{n-1}\right)\right), \emptyset_{2} d\left(g\left(y_{n}\right), g\left(y_{n-1}\right)\right),\right. \\
\left.h_{8} d\left(g\left(x_{n}\right), g\left(x_{n-1}\right)\right), \emptyset_{4} d\left(g\left(z_{n+1}\right), g\left(z_{n}\right)\right), \emptyset_{5} d\left(g\left(x_{n+1}\right), g\left(x_{n}\right)\right)\right\}
\end{gathered}
$$

where, $h_{7(t)}=\max \left\{\varnothing_{1(t)}, \emptyset_{6(t)}\right\}, h_{8(t)}=\max \left\{\varnothing_{3(t)}, \emptyset_{7(t)}\right\}$

$$
\begin{gathered}
d\left(g\left(z_{n+1}\right), g\left(z_{n}\right)\right) \leq \max \left(h_{7} d\left(g\left(z_{n}\right), g\left(z_{n-1}\right)\right), \emptyset_{2} d\left(g\left(y_{n}\right), g\left(y_{n-1}\right)\right),\right. \\
h_{8} d\left(g\left(x_{n}\right), g\left(x_{n-1}\right)\right), \emptyset_{4} d\left(g\left(z_{n+1}\right), g\left(z_{n}\right)\right), \\
\left.\varnothing d\left(g\left(y_{n+1}\right), g\left(y_{n}\right)\right), \emptyset_{5} d\left(g\left(x_{n+1}\right), g_{\left(x_{n}\right)}\right)\right\}
\end{gathered}
$$

Let $\varphi_{(t)}=\max \left\{\varnothing_{(t)}, \varnothing_{1(t)}, \ldots \ldots, \varnothing_{7(t)}, h_{1(t)}, \ldots \ldots, h_{8(t)}\right\}$, then we have $\left.\operatorname{maxim}\left(g\left(x_{n+1}\right), g\left(x_{n}\right)\right), d\left(g\left(y_{n+1}\right), g\left(y_{n}\right)\right), d\left(g\left(z_{n+1}\right), g\left(z_{n}\right)\right)\right\}$

$\leq$ maxiqud $\left(g\left(x_{n}\right), g\left(x_{n-1}\right)\right), \varphi d\left(g\left(y_{n}\right), g\left(y_{n-1}\right)\right)$,

$$
\varphi d\left(g\left(z_{n}\right), g\left(z_{n-1}\right)\right), \varphi d\left(g\left(x_{n+1}\right), g\left(x_{n}\right)\right),
$$

$$
\begin{aligned}
& \left.\varphi d\left(g\left(y_{n+1}\right), g\left(y_{n}\right)\right), \varphi d\left(g\left(z_{n+1}\right), g\left(z_{n}\right)\right)\right\} \\
& <\max \left[\left(d\left(g\left(x_{n}\right), g\left(x_{n-1}\right)\right), d\left(g\left(y_{n}\right),\left(y_{n-1}\right)\right), d\left(g\left(z_{n}\right), g\left(z_{n-1}\right)\right),\right.\right. \\
& \left.d\left(g\left(x_{n+1}\right), g\left(x_{n}\right)\right), d\left(g\left(y_{n+1}\right), g\left(y_{n}\right)\right), d\left(g\left(z_{n+1}\right), g\left(z_{n}\right)\right)\right\}
\end{aligned}
$$

This leads

$$
\begin{aligned}
& \left.\operatorname{maxid}\left(g\left(x_{n+1}\right), g\left(x_{n}\right)\right), d\left(g\left(y_{n+1}\right), g\left(y_{n}\right)\right), d\left(g\left(z_{n+1}\right), g\left(z_{n}\right)\right)\right\} \\
& <\max \left\{d\left(g\left(x_{n}\right), g\left(x_{n-1}\right)\right), d\left(g\left(y_{n}\right), g\left(y_{n-1}\right)\right), d\left(g\left(z_{n}\right), g\left(z_{n-1}\right)\right)\right\}
\end{aligned}
$$

And hence, the equation (2.2) become

$\max$ id $\left.\left(g\left(x_{n+1}\right), g\left(x_{n}\right)\right), d\left(g\left(y_{n+1}\right), g\left(y_{n}\right)\right), d\left(g\left(z_{n+1}\right), g\left(z_{n}\right)\right)\right\}$

$\left.\leq \operatorname{maxim}\left[d\left(g\left(x_{n}\right), g\left(x_{n-1}\right)\right), d\left(g\left(y_{n}\right), g\left(y_{n-1}\right)\right), d\left(g\left(z_{n}\right), g\left(z_{n-1}\right)\right)\right]\right\}$

$\leq \max \left\{\varphi^{2}\left[d\left(g\left(x_{n-1}\right), g\left(x_{n-2}\right)\right), d\left(g\left(y_{n-1}\right), g\left(y_{n-2}\right)\right), d\left(g\left(z_{n-1}\right), g\left(z_{n-2}\right)\right)\right]\right\}$ 


$$
\leq \max \left\{\varphi^{n}\left[d\left(g\left(x_{1}\right), g\left(x_{o}\right)\right), d\left(g\left(y_{1}\right), g\left(y_{o}\right)\right), d\left(g\left(z_{1}\right), g\left(z_{o}\right)\right)\right]\right\}
$$

Butlim $_{n \rightarrow \infty} \max ^{n}\left[d\left(g\left(x_{1}\right), g\left(x_{0}\right)\right), d\left(g\left(y_{1}\right), g\left(y_{0}\right)\right), d\left(g\left(z_{1}\right), g\left(z_{0}\right)\right)\right]=0$

Then, $\forall \epsilon>0 ; \varphi_{(\epsilon)}<\epsilon, \exists n_{\circ} \in N$ such that:

$$
\begin{gathered}
\varphi^{n}\left\{d\left(g\left(x_{1}\right), g\left(x_{\circ}\right)\right), d\left(g\left(y_{1}\right), g\left(y_{o}\right)\right), d\left(g\left(z_{1}\right), g\left(z_{o}\right)\right)\right\}<\epsilon-\varphi_{(\epsilon)} \quad \forall n \geq n_{\circ} \\
\max \left\{d\left(g\left(x_{n+1}\right), g\left(x_{n}\right)\right), d\left(g\left(y_{n+1}\right), g\left(y_{n}\right)\right), d\left(g\left(z_{n+1}\right), g\left(z_{n}\right)\right)\right\} \\
<\epsilon-\varphi_{(\epsilon)}
\end{gathered}
$$

Now, To prove that, $\forall m \geq n \geq n$ 。

$$
\max \left\{d\left(g\left(x_{n}\right), g\left(x_{m}\right)\right), d\left(g\left(y_{n}\right), g\left(y_{m}\right)\right), d\left(g\left(z_{n}\right), g\left(z_{m}\right)\right)\right\}<\epsilon
$$

We will discuss the Cauchy sequence,

i. For $m=n+1$ and by using (2.3) we get (2.4).

ii. Suppose it is if $m=k$, i.e.

$$
\max \left(g d\left(g\left(x_{n}\right), g\left(x_{k}\right)\right), d\left(g\left(y_{n}\right), g\left(y_{k}\right)\right), d\left(g\left(z_{n}\right), g\left(z_{k}\right)\right)\right\}<\epsilon
$$

iii. Now, to prove it is true when $m=k+1$

$$
\begin{aligned}
& d\left(g\left(x_{n}\right), g\left(x_{k+1}\right)\right) \leq d\left(g\left(x_{n}\right), g\left(x_{n+1}\right)\right)+d\left(g\left(x_{n+1}\right), g\left(x_{k+1}\right)\right) \\
& <\epsilon-\varphi_{(\epsilon)}+d f_{1}\left(f_{2}\left(\ldots \ldots\left(f_{n\left(x_{n}, y_{n}, z_{n}\right)}\right) \ldots \ldots\right)\right), f_{1}\left(f_{2}\left(\ldots \ldots\left(f_{n\left(x_{k}, y_{k}, z_{k}\right)}\right) \ldots \ldots\right)\right) \\
& \leq \epsilon-\varphi_{(\epsilon)}+\max _{1} d\left(g\left(x_{n}\right), g\left(x_{k}\right)\right), \emptyset_{2} d\left(g\left(y_{n}\right), g\left(y_{k}\right)\right), \emptyset_{3} d\left(g\left(z_{n}\right), g\left(z_{k}\right)\right) \text {, } \\
& \emptyset_{4} d\left(f_{1}\left(f_{2}\left(\ldots \ldots\left(f_{n\left(x_{n}, y_{n}, z_{n}\right)}\right) \ldots \ldots\right)\right), g\left(x_{n}\right)\right) \text {, } \\
& \emptyset_{5} d\left(f_{1}\left(f_{2}\left(\ldots \ldots\left(f_{n\left(z_{n}, y_{n}, x_{n}\right)}\right) \ldots \ldots\right)\right), g\left(z_{n}\right)\right) \text {, } \\
& \emptyset_{6} d\left(f_{1}\left(f_{2}\left(\ldots \ldots\left(f_{n\left(x_{k}, y_{k}, z_{k}\right)}\right) \ldots \ldots\right)\right), g\left(x_{n}\right)\right) \text {, } \\
& \left.\emptyset_{7} d\left(f_{1}\left(f_{2}\left(\ldots \ldots\left(f_{n\left(z_{k}, y_{k}, x_{k}\right)}\right) \ldots \ldots\right)\right), g\left(z_{n}\right)\right)\right\} \\
& \leq \epsilon-\varphi_{(\epsilon)}+\max \text { (ip }\left(g\left(x_{n}\right), g\left(x_{k}\right)\right), \varphi d\left(g\left(y_{n}\right), g\left(y_{k}\right)\right) \text {, } \\
& \varphi d\left(g\left(z_{n}\right), g\left(z_{k}\right)\right), \varphi d\left(g\left(x_{n+1}\right), g\left(x_{n}\right)\right), \varphi d\left(g\left(z_{n+1}\right), g\left(z_{n}\right)\right) \text {, } \\
& \left.\varphi d\left(g\left(x_{k+1}\right), g\left(x_{k}\right)\right), \varphi d\left(g\left(z_{k+1}\right), g\left(z_{k}\right)\right)\right\} \\
& \leq \epsilon-\varphi_{(\epsilon)} \\
& +\varphi \max \left(g\left(g\left(x_{n}\right), g\left(x_{k}\right)\right), d\left(g\left(y_{n}\right), g\left(y_{k}\right)\right), d\left(g\left(z_{n}\right), g\left(z_{k}\right)\right),\right. \\
& d\left(g\left(x_{n+1}\right), g\left(x_{n}\right)\right),\left(g\left(z_{n+1}\right), g\left(z_{n}\right)\right), d\left(g\left(x_{k+1}\right), g\left(x_{k}\right)\right), \\
& \left.d\left(g\left(z_{k+1}\right), g\left(z_{k}\right)\right)\right\} \\
& \leq \epsilon-\varphi_{(\epsilon)}+\varphi \max \left(g d\left(g\left(x_{n}\right), g\left(x_{k}\right)\right), d\left(g\left(y_{n}\right), g\left(y_{k}\right)\right),\right. \\
& d\left(g\left(z_{n}\right),\left(z_{k}\right)\right), d\left(g\left(x_{n+1}\right),\left(x_{n}\right)\right), d\left(g\left(y_{n+1}\right), g\left(y_{n}\right)\right), \\
& d\left(g\left(z_{n+1}\right), g\left(z_{n}\right)\right), d\left(g\left(x_{k+1}\right), g\left(x_{k}\right)\right), d\left(g\left(y_{k+1}\right), g\left(y_{k}\right)\right), \\
& \left.d\left(g\left(z_{k+1}\right), g\left(z_{k}\right)\right)\right\}
\end{aligned}
$$

$<\epsilon-\varphi_{(\epsilon)}+\varphi \max \left\{\epsilon, \epsilon-\varphi_{(\epsilon)}\right\}$ by (i) and (ii)

$$
<\epsilon-\varphi_{(\epsilon)}+\varphi_{(\epsilon)}=\epsilon
$$

This leads, $d\left(g\left(x_{n}\right), g\left(x_{k+1}\right)\right)<\epsilon$

As the same way, we get $d\left(g\left(y_{n}\right), g\left(y_{k+1}\right)\right)<\epsilon$

and

$$
d\left(g\left(z_{n}\right), g\left(z_{k+1}\right)\right)<\epsilon
$$




\section{And hence,}

$\max \left\{d\left(g\left(x_{n}\right), g\left(x_{k+1}\right)\right), d\left(g\left(y_{n}\right), g\left(y_{k+1}\right)\right), d\left(g\left(z_{n}\right), g\left(z_{k+1}\right)\right)\right\}<\epsilon$.

For all $m \geq n$, (iii) holds, therefor

$$
<g\left(x_{n}\right)>,<g\left(y_{n}\right)>\text { and }<g\left(z_{n}\right)>
$$

are Cauchy sequences in $g(X)$ such that:

- $g\left(x_{1}\right) \leq g\left(x_{2}\right) \leq \cdots \ldots \ldots \leq g\left(x_{n}\right) \leq \cdots \ldots \ldots$ and

$$
g\left(x_{n}\right)=f_{1}\left(f_{2}\left(\ldots \ldots\left(f_{n\left(x_{n-1}, y_{n-1}, z_{n-1}\right)}\right) \ldots \ldots\right)\right)
$$

- $g\left(y_{1}\right) \geq g\left(y_{2}\right) \geq \cdots \ldots \ldots \geq g\left(y_{n}\right) \geq \cdots \ldots \ldots$ and

$$
g\left(y_{n}\right)=f_{1}\left(f_{2}\left(\ldots \ldots\left(f_{n\left(y_{n-1}, x_{n-1}, y_{n-1}\right)}\right) \ldots \ldots\right)\right)
$$

- $g\left(z_{1}\right) \leq g\left(z_{2}\right) \leq \cdots \ldots \ldots \leq g\left(z_{n}\right) \leq \cdots \ldots \ldots$ and

$$
g\left(z_{n}\right)=f_{1}\left(f_{2}\left(\ldots \ldots\left(f_{n\left(z_{n-1}, y_{n-1}, x_{n-1}\right)}\right) \ldots \ldots\right)\right)
$$

But $g(X)$ complete, then there exist $L_{1}, L_{2}, L_{3} \in g(X)$ such that

$g\left(z_{n}\right) \rightarrow L_{3}=g(z) \in g(X)$

$$
\begin{aligned}
& g\left(x_{n}\right) \rightarrow L_{1}=g(x) \in g(X) \\
& g\left(y_{n}\right) \rightarrow L_{2}=g(y) \in g(X)
\end{aligned}
$$

This leads, $\quad g\left(x_{n}\right) \leq g(x), g\left(y_{n}\right) \geq g(y), \quad g\left(z_{n}\right) \leq g(z)$

Now,

$$
\begin{gathered}
d\left(f_{1}\left(f_{2}\left(\ldots \ldots\left(f_{n(x, y, z)}\right) \ldots \ldots\right)\right), g\left(x_{n+1}\right)\right) \\
=d\left(f_{1}\left(f_{2}\left(\ldots \ldots\left(f_{n(x, y, z)}\right) \ldots \ldots\right)\right) f_{1},\left(f_{2}\left(\ldots \ldots\left(f_{n\left(x_{n}, y_{n}, z_{n}\right)}\right) \ldots \ldots\right)\right)\right) \\
\leq \max \left(\emptyset_{1} d\left(g(x), g\left(x_{n}\right)\right), \emptyset_{2} d\left(g(y), g\left(y_{n}\right)\right), \emptyset_{3} d\left(g(z), g\left(\left(z_{n}\right)\right),\right.\right. \\
\emptyset_{4} d\left(f_{1}\left(f_{2}\left(\ldots \ldots\left(f_{n(x, y, z)}\right) \ldots \ldots\right)\right), g(x)\right), \\
\emptyset_{5} d\left(f_{1}\left(f_{2}\left(\ldots \ldots\left(f_{n(z, y, x)}\right) \ldots \ldots\right)\right), g(z)\right), \\
\left.\emptyset_{6} d\left(g\left(x_{n+1}\right), g\left(x_{n}\right)\right), \emptyset_{7} d\left(g\left(z_{n+1}\right), g\left(z_{n}\right)\right)\right\}
\end{gathered}
$$

$\leq \max \left\{\varphi d\left(g(x), g\left(x_{n}\right)\right)\right.$,

$$
\begin{gathered}
\varphi d\left(g(y), g\left(y_{n}\right)\right), \varphi d\left(g(z), g\left(z_{n}\right)\right), \\
\varphi d\left(f_{1}\left(f_{2}\left(\ldots \ldots\left(f_{n(x, y, z)}\right) \ldots \ldots\right)\right), g(x)\right), \\
\varphi d\left(f_{1}\left(f_{2}\left(\ldots \ldots\left(f_{n(z, y, x)}\right) \ldots \ldots\right)\right), g(z)\right), \\
\varphi d\left(f_{1}\left(f_{2}\left(\ldots \ldots\left(f_{n(y, x, y)}\right) \ldots \ldots\right)\right), g(y)\right), \\
\varphi d\left(g\left(x_{n+1}\right), g\left(x_{n}\right)\right), \varphi d\left(g\left(y_{n+1}\right), g\left(y_{n}\right)\right), \\
\left.\varphi d\left(g\left(z_{n+1}\right), g\left(z_{n}\right)\right)\right\}
\end{gathered}
$$

where $\varphi=\max \left\{\varnothing_{1}, \emptyset_{2}, \ldots \ldots, \emptyset_{7}\right\}$

$$
\begin{gathered}
d\left(g\left(y_{n+1}\right), f_{1}\left(f_{2}\left(\ldots \ldots\left(f_{n(y, x, y)}\right) \ldots \ldots\right)\right)\right) \\
=d\left(f_{1}\left(f_{2}\left(\ldots \ldots\left(f_{n\left(y_{n}, x_{n}, y_{n}\right)}\right) \ldots \ldots\right)\right), f_{1}\left(f_{2}\left(\ldots \ldots\left(f_{n(y, x, y)}\right) \ldots \ldots\right)\right)\right) \\
\leq \max \left\{\operatorname{tg} d\left(g\left(y_{n}\right), g(y)\right), \varphi d\left(g\left(x_{n}\right), g(x)\right), \varphi d\left(g\left(y_{n+1}\right), g\left(y_{n}\right)\right),\right. \\
\left.\varphi d\left(f_{1}\left(f_{2}\left(\ldots \ldots\left(f_{n(y, x, y)}\right) \ldots \ldots\right)\right), g(y)\right)\right\}
\end{gathered}
$$




$$
\begin{gathered}
\leq \max \operatorname{tip} d\left(g(x), g\left(x_{n}\right)\right), \varphi d\left(g(y), g\left(y_{n}\right)\right), \varphi d\left(g(z), g\left(z_{n}\right)\right), \\
\varphi d\left(f_{1}\left(f_{2}\left(\ldots \ldots\left(f_{n(x, y, z)}\right) \ldots \ldots\right)\right), g(x)\right), \\
\varphi d\left(f_{1}\left(f_{2}\left(\ldots \ldots\left(f_{n(z, y, x)}\right) \ldots \ldots\right)\right), g(z)\right), \\
\varphi d\left(f_{1}\left(f_{2}\left(\ldots \ldots\left(f_{n(y, x, y)}\right) \ldots \ldots\right)\right), g(y)\right), \\
\varphi d\left(g\left(x_{n+1}\right), g\left(x_{n}\right)\right), \varphi d\left(g\left(y_{n+1}\right), g\left(y_{n}\right)\right), \\
\left.\varphi d\left(g\left(z_{n+1}\right), g\left(z_{n}\right)\right)\right\}
\end{gathered}
$$

As the same way, we get

$$
\begin{aligned}
& d\left(f_{1}\left(f_{2}\left(\ldots \ldots\left(f_{n(x, y, z)}\right) \ldots \ldots\right)\right) f_{1},\left(f_{2}\left(\ldots \ldots\left(f_{n\left(z_{n+1},\right)}\right) \ldots \ldots\right)\right)\right) \\
& \leq \max \text { iq } \varphi d\left(g(x), g\left(x_{n}\right)\right), \varphi d\left(g(y), g\left(y_{n}\right)\right), \varphi d\left(g(z), g\left(z_{n}\right)\right) \text {, } \\
& \varphi d\left(f_{1}\left(f_{2}\left(\ldots \ldots\left(f_{n(x, y, z)}\right) \ldots \ldots\right)\right), g(x)\right), \\
& \varphi d\left(f_{1}\left(f_{2}\left(\ldots \ldots\left(f_{n(z, y, x)}\right) \ldots \ldots\right)\right), g(z)\right) \text {, } \\
& \varphi d\left(f_{1}\left(f_{2}\left(\ldots \ldots\left(f_{n(y, x, y)}\right) \ldots \ldots\right)\right), g(y)\right), \\
& \left.\varphi d\left(g\left(x_{n+1}\right), g\left(x_{n}\right)\right), \varphi d\left(g\left(y_{n+1}\right), g\left(y_{n}\right)\right), \varphi d\left(g\left(z_{n+1}\right), g\left(z_{n}\right)\right)\right\}
\end{aligned}
$$

Now,

$$
\begin{gathered}
\operatorname{maxigd}\left(f_{1}\left(f_{2}\left(\ldots \ldots\left(f_{n(x, y, z)}\right) \ldots \ldots\right)\right), g\left(x_{n+1}\right)\right), \\
d\left(f_{1}\left(f_{2}\left(\ldots \ldots\left(f_{n(y, x, y)}\right) \ldots \ldots\right)\right), g\left(y_{n+1}\right)\right), \\
\left.d\left(f_{1}\left(f_{2}\left(\ldots \ldots\left(f_{n(z, y, x)}\right) \ldots \ldots\right)\right), g\left(z_{n+1}\right)\right)\right\} \\
\leq \max \left(\underline{q} \varphi d\left(g(x), g\left(x_{n}\right)\right), \varphi d\left(g(y), g\left(y_{n}\right)\right), \varphi d\left(g(z), g\left(z_{n}\right)\right),\right. \\
\varphi d\left(f_{1}\left(f_{2}\left(\ldots \ldots\left(f_{n(x, y, z)} \ldots \ldots\right)\right), g(x)\right), \varphi d\left(f_{1}\left(f_{2}\left(\ldots \ldots\left(f_{n(z, y, x)}\right) \ldots \ldots\right)\right), g(z)\right),\right. \\
\varphi d\left(f_{1}\left(f_{2}\left(\ldots \ldots\left(f_{n(y, x, y)}\right) \ldots \ldots\right)\right), g(y)\right), \varphi d\left(g\left(x_{n+1}\right), g\left(x_{n}\right)\right), \\
\left.\varphi d\left(g\left(y_{n+1}\right), g\left(y_{n}\right)\right), \varphi d\left(g\left(z_{n+1}\right), g\left(z_{n}\right)\right)\right\}
\end{gathered}
$$

We claim that

Since, if not

$$
\begin{gathered}
M=\max \left(f_{1}\left(f_{2}\left(\ldots \ldots\left(f_{n(x, y, z)}\right) \ldots \ldots\right)\right), g(x)\right), \\
d\left(f_{1}\left(f_{2}\left(\ldots \ldots\left(f_{n(y, x, y)}\right) \ldots \ldots\right)\right), g(y)\right), \\
\left.d\left(f_{1}\left(f_{2}\left(\ldots \ldots\left(f_{n(z, y, x)}\right) \ldots \ldots\right)\right), g(z)\right)\right\}=0
\end{gathered}
$$

i.e, $M \neq 0$. Let $M=\in>0$

$$
\begin{gathered}
\max \left(f_{1}\left(f_{2}\left(\ldots \ldots\left(f_{n(x, y, z)}\right) \ldots \ldots\right)\right), g(x)\right), \\
d\left(f_{1}\left(f_{2}\left(\ldots \ldots\left(f_{n(y, x, y)}\right) \ldots \ldots\right)\right), g(y)\right), \\
\left.d\left(f_{1}\left(f_{2}\left(\ldots \ldots\left(f_{n(z, y, x)}\right) \ldots \ldots\right)\right), g(z)\right)\right\} \neq 0
\end{gathered}
$$

Since, $d\left(g(x), g\left(x_{n}\right)\right) \rightarrow 0, d\left(g(y), g\left(y_{n}\right)\right) \rightarrow 0, d\left(g(z), g\left(z_{n}\right)\right) \rightarrow 0$

$$
d\left(g\left(x_{n}\right), g\left(x_{n+1}\right)\right) \rightarrow 0, \quad d\left(g\left(y_{n}\right), g\left(y_{n+1}\right)\right) \rightarrow 0 \text { and } d\left(g\left(z_{n}\right), g\left(z_{n+1}\right)\right) \rightarrow 0
$$




\section{Then,}

$$
\begin{aligned}
& \max \left(f_{i}\left(f_{1}\left(f_{2}\left(\ldots \ldots\left(f_{n(x, y, z)}\right) \ldots \ldots\right)\right), g\left(x_{n+1}\right)\right), \quad d\left(f_{1}\left(f_{2}\left(\ldots \ldots\left(f_{n(y, x, y)}\right) \ldots \ldots\right)\right), g\left(y_{n+1}\right)\right), d\left(f_{1}\right.\right. \\
& \leq \max \operatorname{tg} \varphi\left(f_{1}\left(f_{2}\left(\ldots \ldots\left(f_{n(x, y, z)}\right) \ldots \ldots\right)\right), g(x)\right) \text {, } \\
& \varphi d\left(f_{1}\left(f_{2}\left(\ldots \ldots\left(f_{n(z, y, x)}\right) \ldots \ldots\right)\right), g(z)\right), \\
& \left.\varphi d\left(f_{1}\left(f_{2}\left(\ldots \ldots\left(f_{n(y, x, y)}\right) \ldots \ldots\right)\right), g(y)\right)\right\}
\end{aligned}
$$

when $n \rightarrow \infty$, we have

$$
\begin{gathered}
\max \left\{d\left(f_{1}\left(f_{2}\left(\ldots \ldots\left(f_{n(x, y, z)}\right) \ldots \ldots\right)\right), g(x)\right),\right. \\
d\left(f_{1}\left(f_{2}\left(\ldots \ldots\left(f_{n(y, x, y)}\right) \ldots \ldots\right)\right), g(y)\right), \\
\left.d\left(f_{1}\left(f_{2}\left(\ldots \ldots\left(f_{n(z, y, x)}\right) \ldots \ldots\right)\right), g(z)\right)\right\} \\
\leq \max \operatorname{tgd}\left(f_{1}\left(f_{2}\left(\ldots \ldots\left(f_{n(x, y, z)} \ldots \ldots\right)\right), g(x)\right),\right. \\
\operatorname{\varphi d}\left(f_{1}\left(f_{2}\left(\ldots \ldots\left(f_{n(z, y, x)}\right) \ldots \ldots\right)\right), g(z)\right), \\
\left.\varphi d\left(f_{1}\left(f_{2}\left(\ldots \ldots\left(f_{n(y, x, y)}\right) \ldots \ldots\right)\right), g(y)\right)\right\} \\
<\max \left(f_{1}\left(f_{1}\left(f_{2}\left(\ldots \ldots\left(f_{n(x, y, z)}\right) \ldots \ldots\right)\right), g(x)\right),\right. \\
d\left(f_{1}\left(f_{2}\left(\ldots \ldots\left(f_{n(z, y, x)}\right) \ldots \ldots\right)\right), g(z)\right), \\
\left.d\left(f_{1}\left(f_{2}\left(\ldots \ldots\left(f_{n(y, x, y)}\right) \ldots \ldots\right)\right), g(y)\right)\right\}
\end{gathered}
$$

which is a contradiction, and hence $M=0$.That is,

$$
\begin{gathered}
\max \left\{\left(f_{1}\left(f_{2}\left(\ldots \ldots\left(f_{n(x, y, z)}\right) \ldots \ldots\right)\right), g(x)\right),\right. \\
d\left(f_{1}\left(f_{2}\left(\ldots \ldots\left(f_{n(y, x, y)}\right) \ldots \ldots\right)\right), g(y)\right), \\
\left.d\left(f_{1}\left(f_{2}\left(\ldots \ldots\left(f_{n(z, y, x)}\right) \ldots \ldots\right)\right), g(z)\right)\right\}=0
\end{gathered}
$$

This leads

$$
\begin{aligned}
d\left(f_{1}\left(f_{2}\left(\ldots \ldots\left(f_{n(x, y, z)}\right) \ldots \ldots\right)\right), g(x)\right)=0 \\
\\
d\left(f_{1}\left(f_{2}\left(\ldots \ldots\left(f_{n(y, x, y)}\right) \ldots \ldots\right)\right), g(y)\right)=0 \\
\left.d\left(f_{1}\left(f_{2}\left(\ldots \ldots\left(f_{n(z, y, x)}\right) \ldots \ldots\right)\right), g(z)\right)\right\}=0
\end{aligned}
$$

That is $f_{1}\left(f_{2}\left(\ldots \ldots\left(f_{n(x, y, z)}\right) \ldots \ldots\right)\right)=g(x)$

$$
\begin{aligned}
& f_{1}\left(f_{2}\left(\ldots \ldots\left(f_{n(y, x, y)}\right) \ldots \ldots\right)\right)=g(y) \\
& f_{1}\left(f_{2}\left(\ldots \ldots\left(f_{n(z, y, x)}\right) \ldots \ldots\right)\right)=g(z)
\end{aligned}
$$

Therefore, $(x, y, z)$ is public tripled coincidence point of $f_{1}, f_{2}, \ldots \ldots, f_{n}$ and $g$

\section{Corollary(2.2)}

Let $(X, d, \leq)$ be a partially ordered metric space.If $f_{1}, f_{2}, \ldots \ldots, f_{n}: X^{3} \rightarrow X$ and $g: X \rightarrow X$ are mappings lies in $\mathcal{M}$ such that $f_{1}, f_{2}, \ldots \ldots, f_{n}$ having public mixed $g_{-}$monotone property .Suppose that, for all $x, y, z, u, v, w \in X$ with $x \geq u, y \leq v$ and $z \geq w$, 
$d\left(f_{1}\left(f_{2}\left(\ldots \ldots\left(f_{n(x, y, z)}\right) \ldots \ldots\right)\right), f_{1}\left(f_{2}\left(\ldots \ldots\left(f_{n(u, v, w)}\right) \ldots \ldots\right)\right) \leq\right.$

maxis $k_{1} d(g(x), g(u)), k_{2} d(g(y), g(v)), k_{3} d(g(z), g(w))$,

$k_{4} d\left(f_{1}\left(f_{2}\left(\ldots \ldots\left(f_{n(x, y, z)}\right) \ldots \ldots\right)\right), g(x)\right), k_{5} d\left(f_{1}\left(f_{2}\left(\ldots \ldots\left(f_{n(z, y, x)}\right) \ldots \ldots\right)\right), g(z)\right)$,

$\left.k_{6} d\left(f_{1}\left(f_{2}\left(\ldots \ldots\left(f_{n(u, v, w)}\right) \ldots \ldots\right)\right), g(u)\right), k_{7} d\left(f_{1}\left(f_{2}\left(\ldots \ldots\left(f_{n(w, v, u)}\right) \ldots \ldots\right)\right), g(w)\right)\right\}$

where $k_{1}, k_{2}, \ldots \ldots, k_{7} \in[0,1)$ and $\sum_{i=1}^{7} k_{i}<1$.If there exist $x_{\circ}, y_{\circ}, Z_{\circ} \in X$, such that

$g\left(x_{\circ}\right) \leq f_{1}\left(f_{2}\left(\ldots \ldots\left(f_{n\left(x_{0}, y_{0}, z_{0}\right)}\right) \ldots \ldots\right)\right)$

$g\left(y_{\circ}\right) \geq f_{1}\left(f_{2}\left(\ldots \ldots\left(f_{n\left(y_{\circ}, x_{0}, y_{\circ}\right)}\right) \ldots \ldots\right)\right)$ and

$g\left(z_{\circ}\right) \leq f_{1}\left(f_{2}\left(\ldots \ldots\left(f_{n\left(z_{\circ}, y_{\circ}, x_{0}\right)}\right) \ldots \ldots\right)\right)$

Then $, f_{1}, f_{2}, \ldots \ldots, f_{n}$ and $g$ having a public tripled coincidence point

\section{Corollary(2.3)}

Let $(X, d, \leq)$ be a partially ordered metric space.If $f_{1}, f_{2}, \ldots \ldots, f_{n}: X^{3} \rightarrow X$ and $g: X \rightarrow X$ are mappings lies in $\mathcal{M}$ such that $f_{1}, f_{2}, \ldots \ldots, f_{n}$ are public mixed $g_{-}$monotone.Suppose that, for all $x, y, z, u, v, w \in X$ with $x \geq u, y \leq v$ and $z \geq w$,

$d\left(f_{1}\left(f_{2}\left(\ldots \ldots\left(f_{n(x, y, z)}\right) \ldots \ldots\right)\right), f_{1}\left(f_{2}\left(\ldots \ldots\left(f_{n(u, v, w)}\right) \ldots \ldots\right)\right) \leq\right.$

$\max \emptyset\left\{(g(x), g(u)), d\left(g(y), g(v), d\left(g(z), g(w), d\left(f_{1}\left(f_{2}\left(\ldots \ldots\left(f_{n(x, y, z)}\right) \ldots \ldots\right)\right), g(x)\right)\right.\right.\right.$,

$d\left(f_{1}\left(f_{2}\left(\ldots \ldots\left(f_{n(z, y, x)}\right) \ldots \ldots\right)\right), g(z)\right), d\left(f_{1}\left(f_{2}\left(\ldots \ldots\left(f_{n(u, v, w)}\right) \ldots \ldots\right)\right), g(u)\right)$,

$\left.d\left(f_{1}\left(f_{2}\left(\ldots \ldots\left(f_{n(w, v, u)}\right) \ldots \ldots\right)\right), g(w)\right)\right\}$

where $\emptyset \in \Phi$.If there exist $x_{\circ}, y_{\circ}, z_{\circ} \in X$ such that

$g\left(x_{\circ}\right) \leq f_{1}\left(f_{2}\left(\ldots \ldots\left(f_{n\left(x_{0}, y_{0}, z_{0}\right)}\right) \ldots \ldots\right)\right), g\left(y_{\circ}\right) \geq f_{1}\left(f_{2}\left(\ldots \ldots\left(f_{n\left(y_{0}, x_{0}, y_{0}\right)}\right) \ldots \ldots\right)\right)$ and

$g\left(z_{\circ}\right) \leq f_{1}\left(f_{2}\left(\ldots \ldots\left(f_{n\left(z_{\circ}, y_{\circ}, x_{\circ}\right)}\right) \ldots \ldots\right)\right)$. Then $, f_{1}, f_{2}, \ldots \ldots, f_{n}$ and $g$ having $a$ public tripled coincidence point

\section{Corollary(2.4)}

Let $(X, d, \leq)$ be a partially ordered metric space.If $f_{1}, f_{2}, \ldots \ldots, f_{n}: X^{3} \rightarrow X$ and $g: X \rightarrow X$ are mappings lies in $\mathcal{M}$ such that $f_{1}, f_{2}, \ldots \ldots, f_{n}$ having modified $g_{-}$mixed monotone. Suppose that, for all $x, y, z, u, v, w \in X$ with $x \geq u, y \leq v$ and $z \geq w$,

$d\left(f_{1}\left(f_{2}\left(\ldots \ldots\left(f_{n(x, y, z)}\right) \ldots \ldots\right)\right), f_{1}\left(f_{2}\left(\ldots \ldots\left(f_{n(u, v, w)}\right) \ldots \ldots\right)\right) \leq\right.$

$\mathrm{k}$ maxisid $(g(x), g(u)), d(g(y), g(v)), d(g(z), g(w))$,

$d\left(f_{1}\left(f_{2}\left(\ldots \ldots\left(f_{n(x, y, z)}\right) \ldots \ldots\right)\right), g(x)\right), d\left(f_{1}\left(f_{2}\left(\ldots \ldots\left(f_{n(z, y, x)}\right) \ldots \ldots\right)\right), g(z)\right)$

$\left.d\left(f_{1}\left(f_{2}\left(\ldots \ldots\left(f_{n(u, v, w)}\right) \ldots \ldots\right)\right), g(u)\right), d\left(f_{1}\left(f_{2}\left(\ldots \ldots\left(f_{n(w, v, u)}\right) \ldots \ldots\right)\right), g(w)\right)\right\}$

where $k \in[0,1)$

If there exist $x_{\circ}, y_{\circ}, Z_{\circ} \in X$ such that

$g\left(x_{0}\right) \leq f_{1}\left(f_{2}\left(\ldots \ldots\left(f_{n\left(x_{0}, y_{0}, z_{0}\right)}\right) \ldots \ldots\right)\right), g\left(y_{0}\right) \geq f_{1}\left(f_{2}\left(\ldots \ldots\left(f_{n\left(y_{0}, x_{0}, y_{0}\right)}\right) \ldots \ldots\right)\right)$ and

$g\left(z_{\circ}\right) \leq f_{1}\left(f_{2}\left(\ldots \ldots\left(f_{n\left(z_{0}, y_{\circ}, x_{0} \circ\right.}\right) \ldots \ldots\right)\right)$ 
Then $, f_{1}, f_{2}, \ldots \ldots, f_{n}$ and $g$ having a public tripled coincidence point

\section{Corollary(2.5)}

Let $(X, d, \leq)$ be a partially ordered metric space.If $f_{1}, f_{2}, \ldots \ldots, f_{n}: X^{3} \rightarrow X$ and $g: X \rightarrow X$ are mappings lies in $\mathcal{M}$ such that $f_{1}, f_{2}, \ldots \ldots, f_{n}$ having modified $g_{-}$mixed monotone of. Suppose that,for all $x, y, z, u, v, w \in X$ with $x \geq u, y \leq v$ and $z \geq w$,

$$
\begin{aligned}
& d\left(f_{1}\left(f_{2}\left(\ldots \ldots\left(f_{n(x, y, z)}\right) \ldots \ldots\right)\right), f_{1}\left(f_{2}\left(\ldots \ldots\left(f_{n(u, v, w)}\right) \ldots \ldots\right)\right) \leq\right. \\
& k_{1} d(g(x), g(u))+k_{2} d(g(y), g(v))+k_{3} d\left(g_{1}\left(g_{2}\left(\ldots \ldots\left(g_{n(z)}\right) \ldots \ldots\right)\right), g(w)\right)+ \\
& k_{4} d\left(f_{1}\left(f_{2}\left(\ldots \ldots\left(f_{n(x, y, z)}\right) \ldots \ldots\right)\right), g(x)\right)+k_{5} d\left(f_{1}\left(f_{2}\left(\ldots \ldots\left(f_{n(z, y, x)}\right) \ldots \ldots\right)\right), g(z)\right) \\
& \quad+k_{6} d\left(f_{1}\left(f_{2}\left(\ldots \ldots\left(f_{n(u, v, w)}\right) \ldots \ldots\right)\right), g(u)\right) \\
& \quad+k_{7} d\left(f_{1}\left(f_{2}\left(\ldots \ldots\left(f_{n(w, v, u)}\right) \ldots \ldots\right)\right), g(w)\right)
\end{aligned}
$$

where $k_{1}, k_{2}, \ldots \ldots, k_{7} \in[0,1)$ and $\sum_{i=1}^{7} k_{i}<1$

If there exist $x_{\circ}, y_{\circ}, z_{\circ} \in X$ such that

$g\left(x_{\circ}\right) \leq f_{1}\left(f_{2}\left(\ldots \ldots\left(f_{n\left(x_{0}, y_{0}, z_{0}\right)}\right) \ldots \ldots\right)\right), g\left(y_{\circ}\right) \geq f_{1}\left(f_{2}\left(\ldots \ldots\left(f_{n\left(y_{\circ}, x_{0}, y_{0}\right)}\right) \ldots \ldots\right)\right)$ and

$g\left(z_{\circ}\right) \leq f_{1}\left(f_{2}\left(\ldots \ldots\left(f_{n\left(z_{0}, y_{0}, x_{0}\right)}\right) \ldots \ldots\right)\right)$

Then $, f_{1}, f_{2}, \ldots \ldots, f_{n}$ and $g$ having a public tripled coincidence point

Also,you can get others results :

if $g(x)=x$ and $f_{1}, f_{2}, \ldots \ldots, f_{n}$ having public mixed monotone in above results, then we get the public tripled fixed point theorems.

\section{Corollary(2.6)}

Let $(X, d, \leq)$ be a partially ordered metric space.If $f: X^{3} \rightarrow X$ and $g: X \rightarrow X$ are mappings lies in $\mathcal{M}$ such that $f$ having mixed g_monotone property. Suppose that, for all $x, y, z, u, v, w \in X$ with $x \geq u, y \leq v$ and $z \geq w$, $d(f(x, y, z),, f(u, v, w) \leq$

$$
\max _{(1} d(g(x), g(u)), \emptyset_{2} d(g(y), g(v)), \emptyset_{3} d(g(z), g(w)), \emptyset_{4} d(f(x, y, z), g(x)),
$$

$\emptyset_{5} d(f(z, y, x), g(z)), \emptyset_{6} d(f(u, v, w), g(u)), \emptyset_{7} d(f(w, v, u, g(w))\}$

where $\emptyset_{1}, \emptyset_{2}, \ldots \ldots, \emptyset_{7} \in \Phi$

If there exist $x_{\circ}, y_{\circ}, z_{\circ} \in X$ such that

$x_{0} \leq f\left(x_{0}, y_{0}, z_{0}\right), y_{0} \geq f\left(y_{0}, x_{0}, y_{0}\right)$ and

Then,there exists a tripled coincidence fixed point of $f$ and $g$

\section{Corollary(2.7)}

Let $(X, d, \leq)$ be a partially ordered metric space.If $f: X^{3} \rightarrow X$ and $g: X \rightarrow X$ are mappings lies in $\mathcal{M}$ such that $f$ has mixed g_monotone property. Suppose that, for all $x, y, z, u, v, w \in X$ with $x \geq u, y \leq v$ and $z \geq w$, $d(f(x, y, z), f(u, v, w)) \leq$

$$
\text { maxi( } k_{1} d(g(x), g(u)), k_{2} d(g(y),(v)), k_{3} d(g(z), g(w)), k_{4} d(f(x, y, z), g(x)),
$$


$\left.k_{5} d(f(z, y, x), g(z)), k_{6} d(f(u, v, w), g(u)), k_{7} d(f(w, v, u), g(w))\right\}$

where $k_{1}, k_{2}, \ldots \ldots, k_{7} \in[0,1)$ and $\sum_{i=1}^{7} k_{i}<1$

If there exist $x_{\circ}, y_{\circ}, z_{\circ} \in X$ such that

$x_{0} \leq f\left(x_{0}, y_{0}, z_{0}\right), y_{0} \geq f\left(y_{0}, x_{0}, y_{0}\right)$ and $z_{0} \leq f\left(z_{0}, y_{0}, x_{0}\right)$

Then, there exists a tripled coincidence fixed point of $f$ and $g$.

\section{Corollary (2.8)}

Let $(X, d, \leq)$ be a partially ordered metric space. If $f: X^{3} \rightarrow X$ and $g: X \rightarrow X$ are mappings lies in $\mathcal{M}$ such that $f$ has mixed $\mathrm{g}_{-}$monotone property. Suppose that, for all $x, y, z, u, v, w \in X$ with $x \geq u, y \leq v$ and $z \geq w$,

$$
\begin{gathered}
d(f(x, y, z), f(u, v, w) \\
\leq \emptyset(\max \{d(g(x), g(u)), d(g(y), g(v)), d(g(z), g(w)), d(f(z, y, x), g(z)), \\
d(f(x, y, z), g(x)), d(f(u, v, w), g(u)), d(f(w, v, u), g(w))\}), \text { where } \emptyset \in \Phi
\end{gathered}
$$

If there exist $x_{\circ}, y_{\circ}, z_{\circ} \in X$ such that

$x_{0} \leq f\left(x_{0}, y_{0}, z_{0}\right), y_{0} \geq f\left(y_{0}, x_{0}, y_{0}\right)$ and $z_{0} \leq f\left(z_{0}, y_{0}, x_{0}\right)$.

Then, there exists a tripled coincidence fixed point of $f$ and $g$

\section{$\operatorname{Corollary}(2.9)$}

Let $(X, d, \leq)$ be a partially ordered metric space. If $f: X^{3} \rightarrow X$ and $g: X \rightarrow X$ are mappings lies in $\mathcal{M}$ such that $f$ has mixed $\mathrm{g}_{-}$monotone property. Suppose that, for all $x, y, z, u, v, w \in X$ with $x \geq u, y \leq v$ and $z \geq w$,

$d(f(x, y, z), f(u, v, w) \leq$ $k_{1} d(g(x), g(u))+k_{2} d(g(y), g(v))+k_{3} d(g(z), g(w))+k_{4} d(f(x, y, z), g(x))+$ $\left.\left.k_{5} d(f(z, y, x), g(z)), k_{6} d(f(u, v, w), g(u))+k_{7} d(f(w, v, u), g(w))\right\}\right)$,

where $k \in[0,1)$.If there exist $x_{\circ}, y_{\circ}, z_{\circ} \in X$ such that $x_{0} \leq f\left(x_{0}, y_{0}, z_{0}\right), y_{0} \geq f\left(y_{0}, x_{0}, y_{0}\right)$ and $z_{0} \leq f\left(z_{0}, y_{0}, x_{0}\right)$

Then ,there exists a tripled coincidence fixed point of $f$ and $g$.

\section{Corollary(2.10)}

Let $(X, d, \leq)$ be a partially ordered metric space.If $f: X^{3} \rightarrow X$ and $g: X \rightarrow X$ are mappings lies in $\mathcal{M}$ such that $f$ has mixed g-monotone property. Suppose that, for all $x, y, z, u, v, w \in X$ with $x \geq u, y \leq v$ and $z \geq w$, $d(f(x, y, z), f(u, v, w) \leq$ $\mathrm{k}(\max (i d(g(x), g(u)), d(g(y), g(v)), d(g(z), g(w)), d(f(x, y, z), g(x))$, $d(f(u, v, w), g(u)), d(f(w, v, u), g(w))\})$, where $k \in[0,1)$

If there exist $x_{\circ}, y_{\circ}, Z_{\circ} \in X$ such that $x_{0} \leq f\left(x_{0}, y_{0}, z_{0}\right), y_{0} \geq f\left(y_{0}, x_{0}, y_{0}\right)$ and $z_{0} \leq f\left(z_{0}, y_{0}, x_{0}\right)$.

Then,there exists a tripled coincidence fixed point of $f$ and $g$.

\section{References}

[1] ACM. Ran and MCB. Reurings, Afixed point theorem in partially orderedsets and some applications to matrix equations, Proc. Amr .Math. Soc., 132(2004), 1435-1443. 
[2] R.P. Agarwal, M.A. El-Gebeily and D. O'Regan , Generalized contractions in partially ordered metric spaces,Appl. Anal. 87 (2008), (1_8). 1.

[3] I. Altun and H. Simsek, Some fixed point theorems on ordered metric spaces and application, Fixed Point TheoryAppl. 2010 (2010), Article ID 621492, 20 pages. 1.

[4] A. Amini ,Harandi and H. Emami, Afixed point theorem for contraction type maps in partially ordered metric spaces and application to ordinary differential equations, Nonlinear Anal. 72 (2010), 2238_2242. 1.

[5] T.G. Bhaskar and V. Lakshmikantham, Fixed point theorems in partially ordered metric spaces and applications,Nonlinear Anal . 65 (2006),(1379_1393). 1.

[6] B. Choudhury and A. Kundu, A coupled coincidence point result in partially ordered metric spaces for compatiblemappings , Nonlinear Anal. 73 (2010),(2524_2531). 1.

[7] Lj.B. Ciric, N. Cakic, M. Rajovic and J.S.Ume, Monotone generalized nonlinear contractions in partially ordered metric spaces, Fixed point Theory Appl. 2008 (2008), Article ID 131294, 11 pages. 1

[8] Z. Drici, F.A. McRae and J. Vasundhara Devi, Fixed-point theorems in partially ordered metric spaces for operators with PPF dependence, Nonlinear Anal. 67 (2007),(641_647). 1.

[9] J. Harjani and K. Sadarangani, Fixed point theorems for weakly contractive mappings in partially ordered sets,Nonlinear Anal. 71 (2008), 3403_3410. 1.

[10] J. Harjani and K. Sadarangani, Generalized contractions in partially ordered metric spaces and applications toordinary differential equations, Nonlinear Anal. 72 (2010), (1188_1197). 1.

[11] N. Hussain, M.H. Shah and M.A. Kutbi , Coupled coincidence point theorems for nonlinear contractions in partially ordered quasi-metric spaces with a Q-function, Fixed point Theory Appl. 2011 (2011), Article ID 703938, 21 pages.1.

[12] V. Lakshmikantham and Lj.B. Ciric, Coupled fixed point theorems for nonlinear contractions in partially ordered metric spaces, Nonlinear Anal. 70 (2009), 4341_4349. 1.

[13] H.K. Nashine and I. Altun, Fixed point theorems for generalized weakly contractive condition in ordered metric spaces, Fixed point Theory Appl. 2011 (2011), Article ID 132367, 20 pages. 1.

[14] H.K. Nashine and B. Samet, Fixed point results for mappings satisfying $(\psi, \varphi)$-weakly contractive condition in partially ordered metric spaces, Nonlinear Anal. 74 (2011), (2201_2209). 1. [15] H.K. Nashine, B. Samet, C. Vetro, Monotone generalized nonlinear contractions and fixed point theorems inordered metric spaces, Math. Comput. Modelling 54 (2011), (712_720).1.

[16] J.J. Nieto and R.R. Lopez, Contractive mapping theorems in partially ordered sets and applications to ordinary diferential equations, Order 22 (2005),(223_239). 1.

[17] J.J. Nieto and R.R. Lopez, Existence and uniqueness of fixed point in partially ordered sets and applications toordinary differential equations, Acta Math. Sinica, Engl. Ser. 23 (2007), (2205_2212). 1 .

[18] D. O'Regan and A. Petrutel, Fixed point theorems for generalized contractions in ordered metric spaces, J. Math.Anal. Appl. 341 (2008), (1241_1252). 1.

[19] B. Samet, Coupled fixed point theorems for a generalized Meir-Keeler contraction in partially ordered metricspaces, Nonlinear Anal. 72 (2010),( 4508_4517). 1.

[20] W. Shatanawi, Partially ordered cone metric spaces and coupled fixed point results, Comput. Math. Appl. 60(2010),(2508_2515). 1 
[21] Y.Wu, New fixed point theorems and applications of fixed monotone operator, J. Math. Anal. Appl. 341 (2008),(883_893)1

[22] Y. Wu and Z. Liang, Existence and uniqueness of fixed points for mixed monotone operators with applications, Nonlinear Anal. 65 (2006),

(1913_1924)

[23] S. V. Bedre, S. M. Khairnar and B. S. Desale, Hybrid fixed point theorems for M-contraction type maps and applications to functional differential equation, Proceedings of the IMS, Elsevier Science and Technology(October 2013), (390_397).2218

[24] S. V. Bedre, S. M. Khairnar and B. S. Desale, Some fixed point theorems inpartially ordered G-metric spaces and applications to global existence and attractivity results for nonlinear functional integral equations, Proceedings of the ICRTES, Elsevier Science and Technology (November 2013), (467_477).

[25] W. Sintunavarat and P. Kumam and YJ. Cho, Coupled ixed point theorems for nonlinear contractions without mixed monotone property, FixedPoint Theory Appl., 170 (2012).

[26] W. Sintunavarat, S. Radenovi_c, Z. Golubovi_c, P. Kuman, Coupled fixed point theorems for F-invariant set, Appl. Math. Inform. Sci., 7(1)(2013),(247-255).

[27] H. M. Shrivastava, S. V. Bedre, S. M. Khairnar and B. S. Desale, Krasnosel'skii Type Hybrid Fixed Point Theorems and Their Applications toFractional Integral Equations, Abstract and Applied analysis, (accepted).Received: July 15, 2014

[28]V.Berinde, M.Borcut, Tripled Fixed Point Theorems for Contractive Type Mappings in Partially Ordered Metric Space ,Nonlinear Anal .74(2011),(4889_4897)

[29]M.Borcut,V.Berinde ,Tripled coincidence Theorems For contractive Type Mappings in Partially Ordered metric spaces , Appl.Math . Comput .218(2012)5929_5936.

[30].Savitri and N.hooda,"fixed point theorems for mapping having

The mixed monotone property ,Int, Arch .App. Sci. Technol ,Vol 5(2),(2014).(19_23). 